\title{
Estudio de la aplicación de sensores Hall en la medida de desplazamientos micrométricos en sistemas de baja tensión de alimentación
}

\author{
G. Sobreviela, N. Medrano, B. Calvo, S. Celma \\ Group of Electronic Design (GDE) \\ Aragón Institute of Engineering Research (I3A) \\ University of Zaragoza, Pedro Cerbuna 12, 50009, Zaragoza, Spain. \\ Tel.+34-976761240, Fax +34-976762143, e-mail: 579378@celes.unizar.es
}

\begin{abstract}
En la actualidad existen sensores empleados para la medida de desplazamientos micrométricos basados en el campo magnético. Entre ellos los más comunes son los LVDT (Linear Variable Differential Transformer), unos sensores altamente lineales y precisos que son utilizados en aplicaciones que requieren una gran sensibilidad y resolución, como la medida de variaciones de diámetro en plantas asociadas a estrés hídrico. Los sensores LVDT son, por construcción, sistemas relativamente grandes y pesados (ya que la parte activa del sensor es un transformador), y necesitan en general tensiones en el bobinado primario relativamente altas. En la investigación que estamos llevando a cabo hemos sustituido este tipo de sensores por sensores Hall, cuyo tamaño y consumo en potencia es considerablemente inferior que los LVDT, manteniendo una alta sensibilidad como respuesta a variaciones del campo magnético. A su vez, hemos sustituido el núcleo magnético cuyo desplazamiento genera las variaciones del campo magnético por dos discos magnéticos de neodimio $(\mathrm{NdFeB})$ de 0,29 gramos. A día de hoy hemos conseguido que este sistema polarizado con fuente única de $3 \mathrm{~V}$ pueda medir variaciones de desplazamiento con una precisión de $1 \mu \mathrm{m}$. Además, el sistema de acondicionamiento de la señal, constituido por un sistema de amplificación de ventana y un microcontrolador es totalmente programable, lo que facilita su implementación en redes de sensores de bajo consumo y alta autonomía.
\end{abstract}

